\title{
MIMC: Middleware for Identifying \& Mitigating Congestion Level in Hybrid Mobile Adhoc Network
}

\author{
P. G. Sunitha Hiremath \\ Assc Prof \\ Dept. of Information Science \& Engg., BVBCET, Hubli, \\ India
}

\author{
C.V. Guru Rao \\ Director of Evaluation, \\ S.R. Engg. College, Warangal, \\ India
}

\begin{abstract}
Adoption of middleware system to solve the congestion problem in mobile ad-hoc network is few to find in the existing system. Research gap is found as existing congestion control mechanism in MANET doesn't use middleware design and existing middleware system were never investigated for its applicability in congestion control over the mobile ad-hoc network. Therefore, we introduce a novel middleware system called as MIMC or Middleware for Identifying and Mitigating Congestion in Hybrid Mobile Adhoc Network. MIMC is also equipped with novel traffic modeling using rule-based control matrix that not only provides a better scenario of congestion but also assists in decision making for routing, which the existing techniques fails. This paper discusses the algorithms, result discussion on multiple scenarios to show MIMC perform better congestion control as compared to existing techniques.
\end{abstract}

Keyword-Middleware; Congestion Control; Traffic Management; Hybrid Mobile Adhoc network

\section{INTRODUCTION}

The mobile ad-hoc network has played a huge contribution in the area of the wireless ad-hoc network, and it has been a point of major research area since last decades in wireless networking. Till date, there has been researching on multiple problems in mobile ad-hoc network e.g. routing issues [1][2], energy issues [2], security issues [3], load balancing issues [4], congestion control issues [5], etc. Out of all the problems, routing and security have attained much attention. We will like to discuss the congestion problems in the mobile ad-hoc network that occurs due to superfluous number of data bigger than channel capacity. An adverse effect of congestion is a loss of data packets, intermittent links, interference, etc. [6]. Hence, it is highly essential to perform controlling of congestion in a dynamic topology of the mobile ad-hoc network. One of the biggest problems with the existing congestion control protocol is non-consideration of routing schema [7]. A closer look at the trends of research shows that majority of the studies towards congestion control is focused on homogeneous network and very less focus is laid on to a heterogeneous network. This evidence can also be found by observing less number of work being carried out in designing middleware system in the mobile ad-hoc network. The evolution of middleware is not new, but they are more involved in the theoretical study and less in practical implementation. Problems of middleware to be more focused on resource management is seen in theory and very less on implementation papers. A middleware must be able to configure multiple forms of resources in many ways [8][9]. However, usage of middleware is not that much clear in the research area of the mobile ad-hoc network. Till date, there is no discussion of any middleware systems over a mobile ad-hoc network that can perform identification of the state of traffic congestion and provides a solution to mitigate it. Hence, this paper presents a novel middleware system that can perform identification of congestion in the mobile ad-hoc network. The design principle of the proposed middleware system is meant to achieve i) seamless and cost-effective monitoring of traffic condition for mobile ad-hoc network and its applications (e.g. vehicular network), ii) assists in relaying information about the congestion level of traffic in the entire network, iii) less overhead in message dissemination, etc. The paper presents a costeffective technique of middleware services which provides interoperability, energy efficiency, as well as congestion control mechanism in the hybrid mobile ad-hoc network. Section II discusses the background of the study where the discussion of the recent system of traffic congestion control in the mobile ad-hoc network is carried out followed by brief discussion of problems in Section III. Section IV discusses the contribution of the proposed system regarding adopted research methodology. Section $\mathrm{V}$ discusses the algorithm discussion followed by result discussion in Section VI. Finally, the findings of the proposed study are summarized in Section VII.

\section{Related Work}

This section discusses about various significant studies conducted towards normalizing the traffic behavior in hybrid mobile adhoc network. The primary motive of this section is to showcase the research papers towards addressing the problems of traffic management using middleware-based solution for hybrid mobile adhoc network.

Vadivel and Bhaskaran [10] has presented a recent technique of formulating congestion-free routes as well as to the study has also introduced a reliable routing in the mobile adhoc network. The author has used a mechanism of disjoint path construction for balancing the traffic load. The study outcome was found to posses better communication performance. Study towards addressing congestion problem was also discussed by Sirajuddin et al. [11], where the authors has used a TCP-based congestion control management over a mobile ad-hoc network. The technique mainly forms a network followed by propagation of congestion status and finally by route maintenance depending on the status of traffic load. The study outcome was compared with conventional AODV on packet delivery ratio and routing overhead. 
Pashchenko et al. [12] has presented a model that is designed for cloud model for networking. However, the entire modelling was carried out using the concept of middleware in mobile ad-hoc network. The discussion laid by the author is highly helpful as all the discussed formal models in this research papers can be readily used in any form of wireless network for traffic management including QoS as well as security. The study carried out by Chen et al. [13] has introduced a cross-layered architecture incorporated on the integrated scheme of congestion management and QoS scheduling in a wireless network which supports multihop. The technique uses Differentiated Queuing Service along with partial TCP services for this purpose. The study outcome was testified for reduced delay and increased delivery ratio.

Sreenivas et al. [14] has presented an approach that can perform controlling of traffic congestion over the mobile ad-hoc network after enhancing TCP. According to this technique, the significant information of the network status is identified by destination node that further transmits to the source node in the form of response. The study outcome was evaluated to posses better throughput and delay performance. Greco et al. [15] has presented a technique to overcome the problem of latency considering the case study of multimedia streaming over the mobile ad-hoc network. The technique uses a cross layer-based approach where coding of multimedia is carried over MAC layer followed by optimization over the state of congestion in the mobile ad-hoc network. The author has developed a distortion model powered by sophisticated mathematical modelling using graph theory to perform congestion identification and mitigation. The study outcome was evaluated on relative frequency over increasing delay and probability density over PSNR. Bhaduria and Sharma [16] has presented a framework that can control congestion in a mobile ad-hoc network with the aid of agent-based services. The technique allows selection of less-congested route

Qin et al. [17] has developed a middleware approach for catering up the need of the heterogeneous networking environment, which is based on the self-intelligence building process to accomplish better communication performance. The system uses observe-analyze-adapt methodology for developing the formal node model. Study towards the evolution of middleware was seen in the work of Kamisinski et al. [18]. The technique was meant for sensor nodes for processing complex information entrapped by a sensor using distributed middleware system. Testified over multiple mobility environments, the technique allows dynamic selection of routing algorithm depending on the need of applications. Another study of middle concerning about traffic management was carried out by Denker et al. [19]. The study mainly focuses on cyber-physical systems and highlights about its dependencies. Pease [20] has developed a middleware framework called as ROAM which intends to support energy communication using cross layered approach in mobile ad-hoc network.

The study uses cross-layer approach to identify the new route and performs blacklisting of the path which is inflicted with channel fading problem. Another middleware approach was presented by Lopez et al. [21] where the author has presented a technique to maintains the portability of the routing protocols in mobile adhoc network. The technique has used multicast protocol design to develop a group communication system in mobile adhoc network. The study outcome was evaluated on a real test bed on some the message being used for transmission. Liu [22] has presented a study of middleware system for a wireless network using context-based factors. The prime purpose of this system is to present a technique with better supportability of distributed synchronization and dynamic reconfiguration.

Hence, it can be seen that there are various studies car-ried out for congestion control as well as the various design of middleware to upgrade the communication performance of mobile adhoc network. Each study has its beneficial factor regarding communication performance and problems being discussed in the research papers. However, these studies are also associated with certain loopholes when it comes to congestion control over hybrid the mobile ad-hoc network using middlewarebased approach. The next section highlights significant points of limitations and justifies the evolution of problem statement.

\section{PROBLEM DESCRIPTION}

This section discusses the problems being identified after reviewing the existing system discussed in prior section.

\section{A. Less Applicability of existing Congestion Control:}

Although, there is a certain level of work being carried out addressing congestion issues over heterogeneous mobile adhoc network e.g. [23], none of them has addressed the interoperable part of it. Hence, heterogeneous network without the inclusion of interoperability is not the purely heterogeneous network. Hence, such algorithms are less likely to be effective when implemented over the real-world environment. The existing techniques of congestion control are more inclined towards homogeneous network and very less towards heterogeneous networks.

\section{B. Frequent usage of Cross Layer Approach:}

It is highly essential that the design approach of a middleware must has the knowledge of the topology to use for a multihop communication system, and it maintains its efficiency. Hence, adoption of cross-layer approach assists over kernel space in communicating with networking protocols. This approaches, therefore, violates the stringent layering mechanism of the network stack in the mobile ad-hoc network that obstructs the true performance of middleware services.

\section{Lew focus on hybrid networks:}

The majority of the study towards traffic management, congestion control, load balancing task scheduling, etc. are either focused on reactive or proactive routing protocols in the mobile ad-hoc network. However, similar area of research on hybrid routing methodology was less considered. There exists certain few research works focusing on hybrid routing e.g. [24][25], but they are fewer standards in nature or has a very limited scope of future enhancement. Hence, study on congestion control is quite a few to find on hybrid networks.

A closer look at the problem identification points says that although there has been work carried out for congestion control in mobile ad-hoc network, there is a bigger trade-off between congestion control and adoption of the hybrid protocol as well as middleware design. Middleware design can act as a robust 
bridge of interoperability and thereby can act upon heterogeneous mobile ad-hoc network. However, in the past, there is almost no research manuscript being found for addressing the problem of congestion using middleware-based approach. It is going to be completely a new arena of research, whereby hybrid network adoption will be the first to be tested for congestion control using middleware design. Therefore, the problem statement of the proposed system can be represented as -"It is a novel and challenging task to develop a modelling of middleware system that can carry out congestion control mechanism over hybrid network to attain better communication performance."

\section{PROPOSED MIMC Methodology}

The proposed study is an extension of our prior studies where we has introduced two different characteristics on our middleware design i.e. interoperability and energy efficiency. Our first model MERAM [26] is focused on incorporating interoperability in its middleware design whereas the second model MEEM [27] is more focused on developing energy efficient features. The proposed model MIMC aims at incorporating congestion identification and mitigation characteristics for hybrid mobile ad-hoc network.

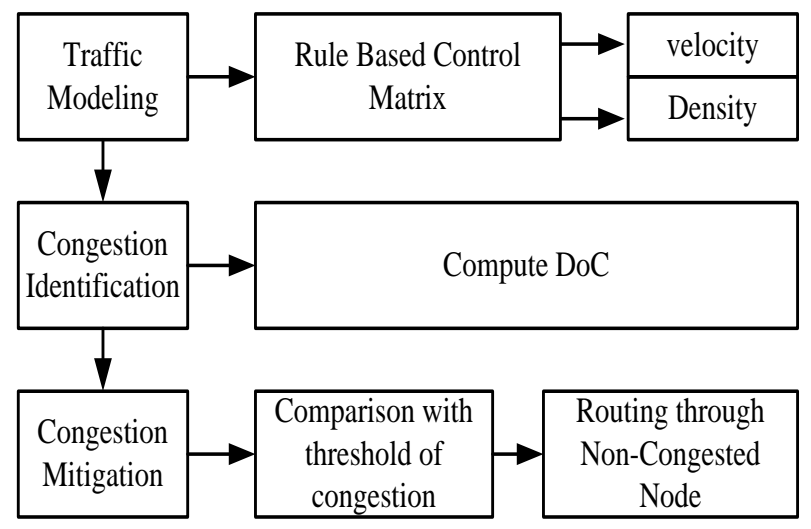

Fig. 1. Proposed Schema of MIMC

The proposed study is developed using analytical research methodology where the mechanism of MIMC is divided into three core modules i.e. traffic modelling, congestion identification, and congestion mitigation. Traffic modelling is carried out using multi-valued logic which provides the better shape of hybrid mobile ad-hoc network on real-world applications using mobile nodes. The system uses pre-defined ruleset called as Rule Based Control Matrix which gives inferences based on velocity and density of node. The second module is about exploring the presence of congestion in neighbor nodes. MIMC doesn't attempt to spend its resources exploring the source of congestion rather it uses its resources just to find the alternative routes. Hence, it is much cost effective and provides a better response time for exploring non-congested routes using a new term called as Degree of Congestion or DoC. The last step is to perform mitigation of congestion, which will mean filtering the message that estimates the congestion and compares it with a threshold. An elaborated information about its implementation is discussed in next section.

\section{ALGORITHM IMPLEMENTATION}

This section discusses the algorithm design and implementation that was used in proposed middleware system in the hybrid mobile ad-hoc network. It should be noted that the proposed middleware system is meant to carry out following operation i.e. i) traffic modelling, ii) congestion identification and iii) congestion control. Following are the detailed information about it.

a) Traffic Modelling: - MIMC make use of node-tonode communication system to perform traffic modelling along with communication. The algorithm to develop traffic modelling takes the input of $\mathrm{v}$ (velocity), $\mathrm{p}$ (position), and $\mathrm{t}$ (time interval), which after processing yields simulated a version of the traffic in hybrid mobile ad-hoc network. For this purpose, we develop a simple control message msg which will carry velocity ( $v$ ) and positional (p) information of source node and should keep on exchanging with its neighbor nodes after a specific interval of time $t$.

\begin{tabular}{|c|}
\hline $\begin{array}{l}\text { Algorithm for Traffic Modelling } \\
\text { Input: } v \text { (velocity), } p \text { (position), } t \text { (time interval), } d \text { (density of nodes), RBCM (Rule } \\
\text { Based Control Matrix) } \\
\text { Output: traff (traffic) } \\
\text { Start } \\
\text { 1. Init v, p, t } \\
\text { 2. Exchange beacons after every t-sec } \\
\text { i(msg[v,p]) } \Leftrightarrow j(m s g[v, p]) \\
\text { 3. } v=\sum_{x=1}^{4} v v_{x} \& d=\sum_{y=1}^{4} d_{y} \\
\text { 4. } f(\mathrm{RBCM}) \leftarrow[\mathrm{v}, \mathrm{d}] \\
\text { 5. traff } \rightarrow \operatorname{simu}{ }_{s c o p e}[f(\mathrm{RBCM})] \\
\text { End }\end{array}$ \\
\hline
\end{tabular}

Fig. 2. Algorithm for Traffic Modelling

To overcome the problem of topological dynamicity of the mobile nodes, we will use a novel rule-based mechanism based on simple mathematical logic to carry out traffic modelling. Our traffic modeling is not the simply distribution of nodes with mobility, but we emphasize more on the distinctive behavior of a node by monitoring the types of beacons msg being relayed by the nodes. For this purpose, we develop a simple matrix called as RBCM or Rule Based Control Matrix which discretely defines the various logical inference of the ruleset for velocity (v) and density (d). The algorithm exchanges of the control message among the communicating node (Line-2), which allows the mobile nodes to get updates status of the congestion level. The system considers 4 different states of velocity (fast, normal, slow, and very slow) whereas there are four discrete states for node density (i.e. less, medium, high, and very high) (Line-3). 
TABLE I. FORMATION OF RULE-BASED CONTROL MATRIX

\begin{tabular}{|c|l|l|l|l|l|}
\hline \multirow{2}{*}{} & \multicolumn{4}{|c|}{ Degree of Congestion (DoC) } \\
\cline { 3 - 6 } & \multicolumn{4}{|c|}{ Velocity of mobile node } \\
\cline { 2 - 6 } & Fast & Normal & Slow & Very Slow \\
\cline { 2 - 5 } & & Free & Free & Free & Slight \\
\cline { 2 - 5 } & Less & Free & Slight & Slight & Moderate \\
\cline { 2 - 5 } & Medium & Free & Slight & Moderate & Moderate \\
\cline { 2 - 6 } & High & Slight & Moderate & Moderate & Severe \\
\cline { 2 - 5 } & $\begin{array}{l}\text { Very } \\
\text { High }\end{array}$ & & & & \\
\hline
\end{tabular}

A function is developed for RBCM (Rule-Based Control Matrix) whose matrix formation is tabulated in Table.1, which creates a simple inference rules based on the types of velocity and node density as input arguments. The algorithm computes velocity from a distance traveled at a specific interval of time. Computation of node density is quite a difficult task. Depending on the number of the controlled messages received, each mobile node computes the total number of neighbor nodes at that period as the node density. The mobile nodes estimate the velocity factor by dividing instantaneous velocity with velocity limit assigned for the path. Hence, it is possible to calculate the highest number of mobile nodes in every path using a range of transmission. Hence, for a given section of a route, every mobile node can calculate the number of mobile nodes in that particular route segment by the ratio of total existing neighbor nodes in route section with the highest number of mobile nodes that can be accommodated in that particular route. The advantage of this traffic modelling is its simple inference mechanism that allows more flexibility of investigating middleware system for hybrid mobile ad-hoc network.

b) Congestion Identification: - The proposed MIMC adopts node-to-node interaction system to find the Degree of Congestion (DoC). The prior traffic model allows confirming this degree of congestion using RBCM. Therefore, the control message used msg can also be termed as a message for identifying (or estimating) congestion degree. The proposed mechanism initially finds the size of the control message msg with predefined threshold used for congestion i.e. Th (Line-1). The algorithm computes Degree of Congestion using neighboring nodes $(\mathrm{NN})$ and frequency intervals (FI) (Line-2). The mobile node that computes DoC is considered to be mobile reference node and is used for computation of DoC. If the Degree of Congestion is found to be more than the threshold (Line-3) than it represents an event of congestion, however, it doesn't give a clear view of its location. Moreover, MIMC do not involve route acknowledgment messages to state confirmation of the message delivery. Further, overhead is minimized by lowering the frequency (Line-5). The algorithm than continues its search for all the nodes whose Degree of Congestion level is more than the threshold to identify the location of those nodes. Only the nodes with a higher value of DoC will be considered for performing retransmission process. However, if any mobile nodes pass within the transmission range of this node than it halts its transmission (Line-6-9).

Algorithm for Congestion Identification
Input: $\mathrm{DoC}$ (Degree of Congestion), $\mathrm{F}_{\mathrm{I}}$ (frequency intervals), $\mathrm{N}_{\mathrm{N}}$ (Number of neigh-
bor nodes), $\mathrm{E}_{\mathrm{D}}$ (Effective Distance), $\mathrm{T}_{\mathrm{r}}$ (Transmission range), $\mathrm{W}_{\text {node }}$ (wavelength of
node), $\mathrm{f}($ frequency), $\mathrm{Th}$ (Threshold)
Output: Congestion identification
Start
1. $\mathrm{msg} \rightarrow$ size(msg)
2. DoC $=\left[\mathrm{F}_{\mathrm{I}}, \mathrm{N}_{\mathrm{N}}\right]$
3. if DoC $>\mathrm{Th}$
4. Congestion Identified
5. $\mathrm{f}(\mathrm{f}-0.1)$
6. If node(DoC $>\mathrm{Th}))$
7. node $\rightarrow$ retrans
8. else
9. Permit routing
10. compute relay distance of nodes
$E_{D}=T_{r}-\left[W_{\text {node }}\right]$
11. node $\rightarrow$ route( $\left.\mathrm{E}_{\mathrm{D}}\right)$
End

Fig. 3. Algorithm for the Congestion Identification

Fig.4 highlights the schema that has been used in this algorithm.

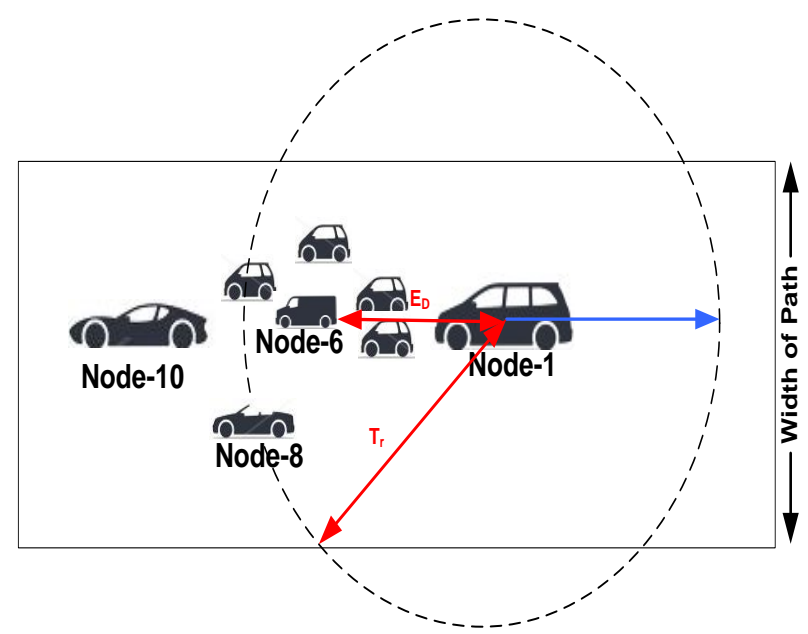

Fig. 4. Schema of routing used in the proposed system

Finally, the algorithm computes effective distance ED (in meters) which is calculated by subtracting transmission range (Tr) with a wavelength of the node (W node)(in meters) (Line10). The wavelength can be further calculated by highest velocity permissible (in meter per second) on that specific path divided by the frequency of transmission of control message (in Hertz). Fig. 4 showcase a scenario which represents node- 8 is at more distance from node- 1 and hence node- 8 will have lesser probability to relay the control message for congestion identification. However, node- 6 can be considered to be suitable relay node and will calculate effective distance $E_{D}$. 
c) Congestion Mitigation:- After the degree of congestion along with location is identified, the next task is to perform mitigation of the congestion. The term mitigation will mean a mechanism to continue communication through alternative routes. From a review of the literature, it has been already seen that existing middleware design of congestion control doesn't consider decision making to be involved. Therefore, we address this problem by formulating an algorithm that supports collaborative networks in hybrid mobile ad-hoc network. The primary target of this algorithm is to minimize the duration of mobile nodes on the path and thereby to recommend the changes in the routes for an alternative solution to congestion.

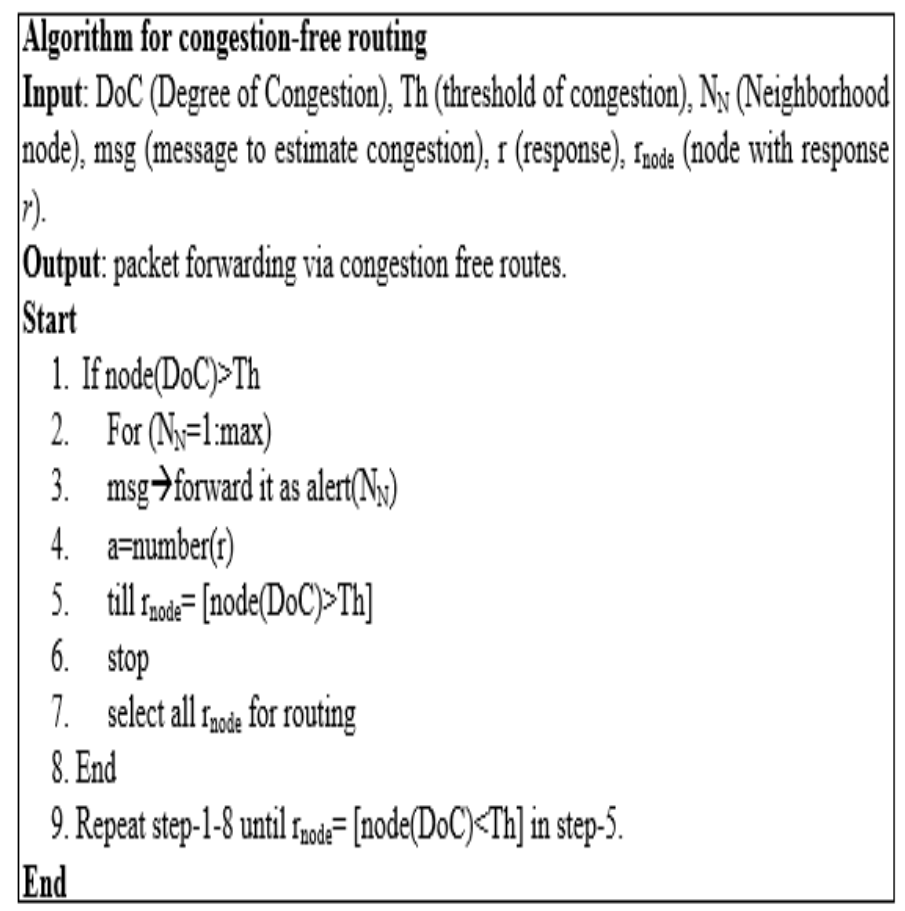

Fig. 5. Algorithm for congestion-free routing

The algorithm checks for a degree of congestion of the nodes to be more than the threshold level of congestion (Line1 ), which corresponds to the state of congestion. In such case, the algorithm looks for all possible neighbor nodes and the search for a chain of neighbor nodes continues (Line-2). In this search, only nodes with a lesser value of the degree of congestion are recorded to be a reliable path for undertaking routing decision. However, for better search optimization, we terminate our search for efficient node exactly for the node with more value of the degree of congestion (Line-5). For all the neighboring nodes whose degree of congestion is within the lower limit of Threshold (Line-9), the control message is transformed to alert message emphasizing on relaying the information that source node is already detected with congestion and hence an alternative congestion-free route must be found.
TABLE II. NOTATION USED IN ALGORITHM DESIGN

\begin{tabular}{|l|l|}
\hline Notation & Meaning \\
\hline$v$ & velocity \\
\hline$p$ & Position \\
\hline$t$ & time interval \\
\hline$d$ & density of nodes \\
\hline$R B C M$ & Rule Based Control Matrix \\
\hline traff & traffic \\
\hline DoC & Degree of Congestion \\
\hline $\mathrm{F}_{\mathrm{I}}$ & Frequency Intervals \\
\hline $\mathrm{N}_{\mathrm{N}}$ & Number of neighbor nodes \\
\hline $\mathrm{E}_{\mathrm{D}}$ & Effective Distance \\
\hline $\mathrm{T}_{\mathrm{r}}$ & Transmission range \\
\hline $\mathrm{W}_{\text {node }}$ & wavelength of node \\
\hline $\mathrm{F}$ & frequency \\
\hline $\mathrm{Th}$ & Threshold \\
\hline $\mathrm{msg}$ & message to estimate congestion \\
\hline $\mathrm{R}$ & response \\
\hline $\mathrm{r}_{\text {node }}$ & node with response $r$ \\
\hline
\end{tabular}

\section{RESUlt DISCUSSION}

The implementation of the proposed algorithm is carried out in Matlab, where the target was to investigate the factors that have a significant impact on the congestion. A completely new simulator is developed for this purpose considering random mobility factor. We have also taken a case study of a vehicular ad-hoc network to investigate the validity of the proposed concept of middleware system of MIMC. The complete analysis of the results is an outcome of node-to-node communication system considered over the simulation parameters tabulated below:

TABLE III. SimULATION PARAMETERS

\begin{tabular}{|l|l|}
\hline Number of Mobile Nodes & $500-1000$ \\
\hline Simulation Time & 600 Seconds \\
\hline Minimum velocity & 5 meter per second \\
\hline Maximum velocity & 100 meter per seconds \\
\hline Communication range & 200 meters \\
\hline Simulation Rounds & $1000-7000$ \\
\hline \\
\hline
\end{tabular}

(a) Section of Path with mobile nodes

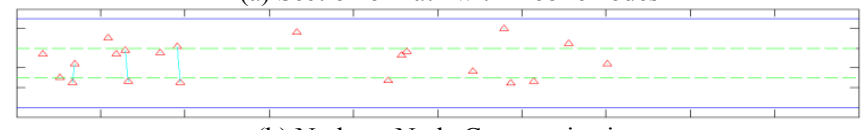

(b) Node-to-Node Communication

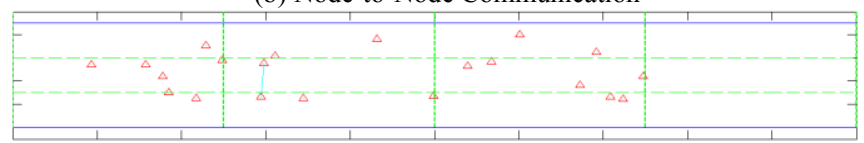

(c) Mobility and Communication in present of obstruction

Fig. 6. MIMC Simulations of Mobile Nodes 
Fig.6 shows the visual outcomes of the simulation carried out in MIMC for the mobile node (Red triangle). A simulation allows a simple segment of the route with multiple lane system (dotted green) as seen in Fig. 6(a). Depending upon the input of simulation parameters (especially the node velocity and transmission range), the node-to-node communication starts between the nodes residing in multiple lanes. The communication link is highlighted as blue line between three pairs of nodes in Fig.6. (b). We also add obstruction in the traffic (shown in vertical dotted green line), which mimics the speed bumpers on the real-word road (Fig.6(c)). It may also represents the traffic signal which we get to see in urban vehicular adhoc networks to some extent. Inclusion of obstruction is meant for reducing the initialized velocity of the mobile node. Although, we initialize minimum and maximum velocity of the node, such initialization is than randomized to total number of mobile nodes considered in the simulation area. This is done in order to incorporate dynamic topology of mobile adhoc network; however we have restricted the dynamicity by letting the mobile nodes to go in one direction of the path. Hence, directionality of the mobile node is fixed in order to check the consistency of the performance of the proposed middleware system with respect to congestion control in real-world road.

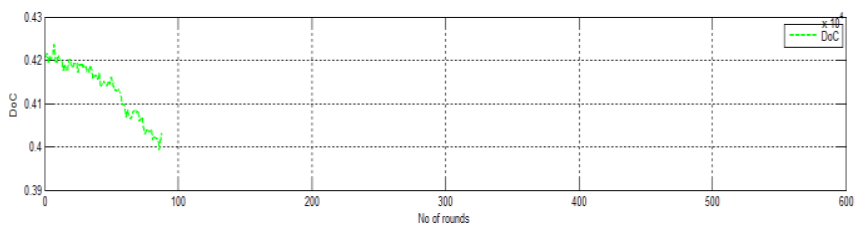

(a) Trend of DoC in MIMC during $100^{\text {th }}$ test simulation rounds

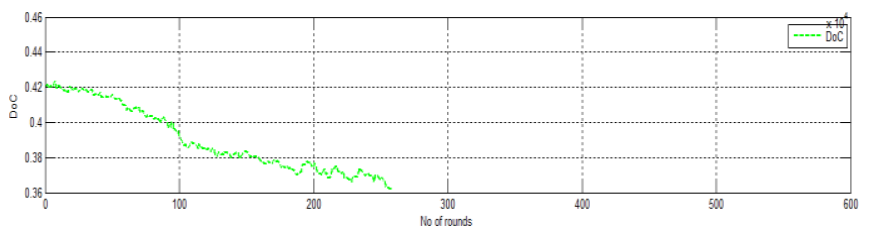

(b) Trend of DoC in MIMC during $250^{\text {th }}$ test simulation rounds

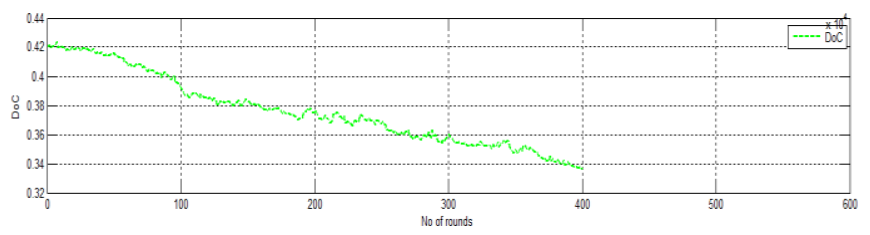

(c) Trend of DoC in MIMC during $400^{\text {th }}$ test simulation rounds

Fig. 7. Progressive DoC Observation in MIMC Simulation
The frequently used existing simulators e.g. NS2, OM$\mathrm{NeT}++$, OPNET, etc. does show the simulation of the considered environment, but cannot show the live and dynamic generation of graphical trends during the simulation progress. Dynamic investigation of graphical trend becomes an important operation especially when the investigational rounds are quite high and when there is a need to observe the live trends. We did this feature in our MIMC because we want the implication of MIMC to be more on online result analysis and not on offline result analysis. Hence, this was only possible by Matlab by designing its method and hence highly customizable. Fig.7. (a)-(c) shows the progress being made during each round of simulation. A closer look into the graphical trend will tell that value of degree of congestion i.e. DoC is found to progress in decreasing order.

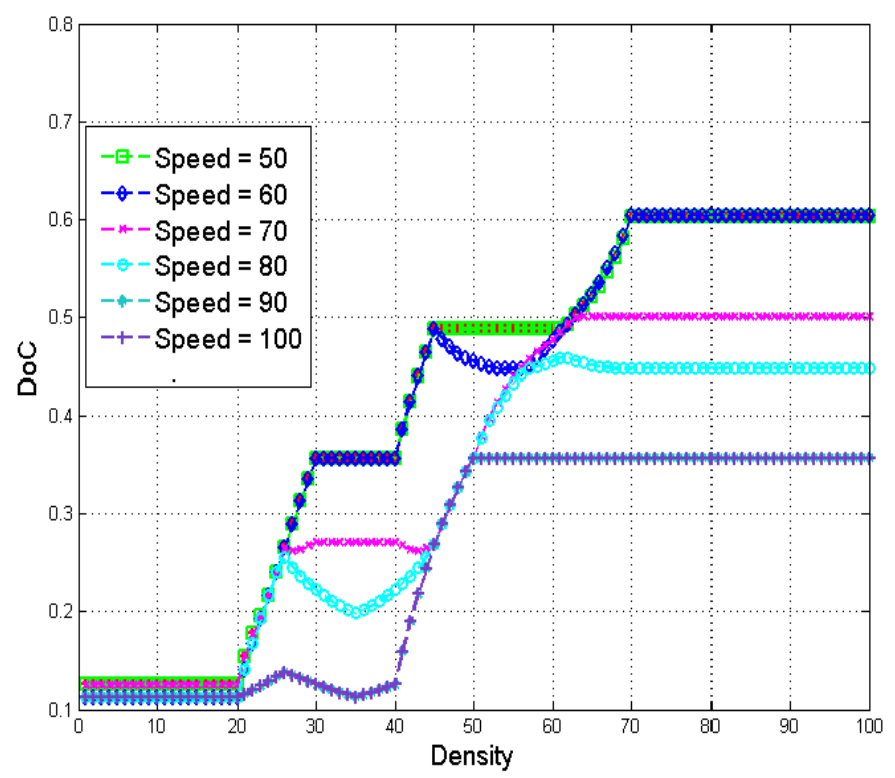

Fig. 8. Impact of Velocity over DoC

Fig. 8 shows the impact of the velocity over the DoC on increasing values of density of nodes. It is already known that increase in density of nodes will eventually increase in DoC value, but there are multiple mobility scenarios that require closer observation. Initially, we initiated the velocity with $v=50$ ms- 1 and kept it constantly increasing with $10 \mathrm{~ms}-1$ till it reaches $100 \mathrm{~ms}-1$. The outcome shows that MIMC is capable of retaining DoC to lower values even if the speed of the velocity is increased, which is in agreement that increased velocity and reduced density is ideal state of non-congestion and vice-versa. 

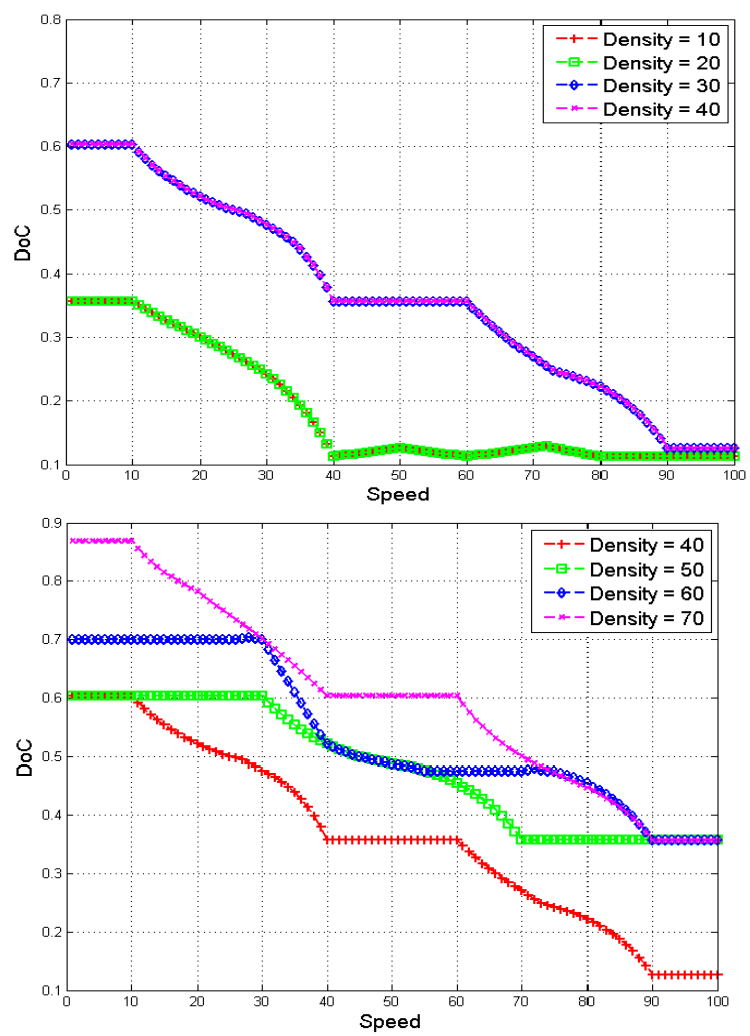

(a) Density $\rightarrow[10-40$ nodes $]$

(b) Density $\rightarrow[40-50$ nodes $]$
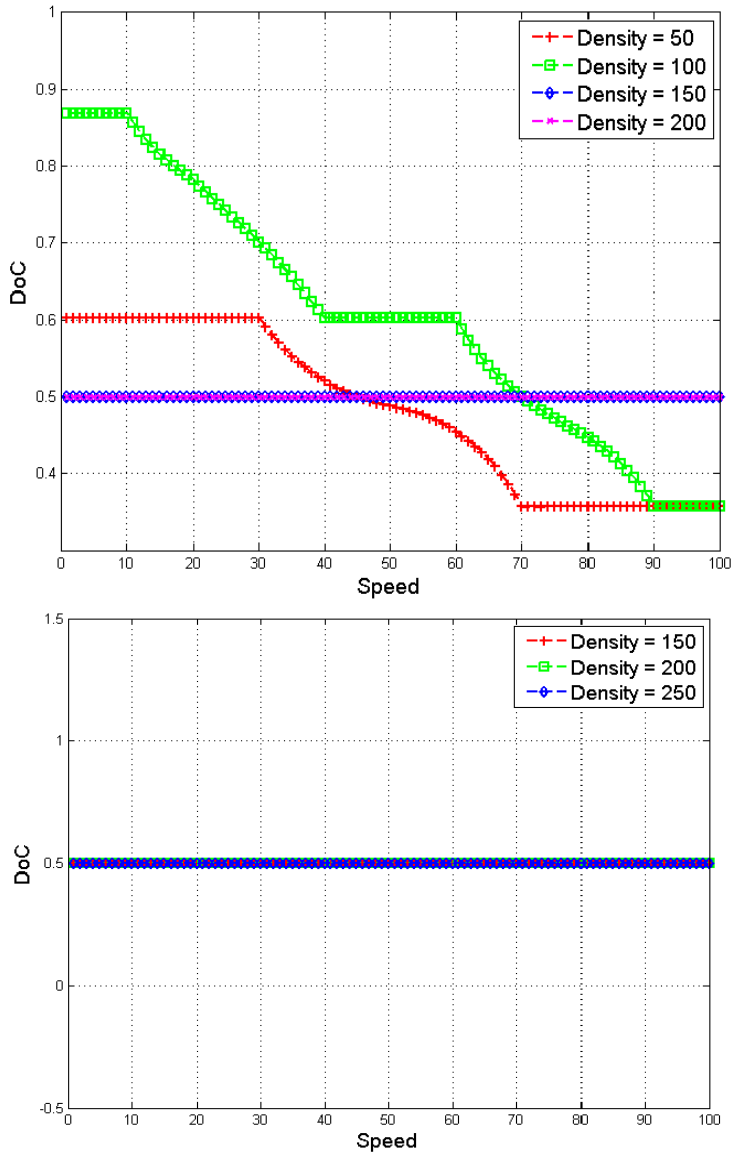

(c) Density $\rightarrow[50-200$ nodes $]$

(b) Density $\rightarrow[150-250$ nodes $]$
Fig.9 showcases the impact of the node density over the value of the degree of congestion. Anticipating lower value of DoC is quite imperative for the lower value of node density. However, we will like to check the upper limit of it. Hence, the initial investigation with density maintained between 10-40 nodes show a normal decreasing trend of DoC as shown in Fig. 9(a). However, when the density is increased from 50-70 nodes, we find a bit of variation in the DoC trend. It shows increasing values of node density will increase DoC too (Fig. 9(b)). Further, density is again increased from 50 to 200 nodes to find that density of 50,100, and 150 nodes as a distinctive trend. At the same time, we find a linear behavior for density maintained to be 150 and 200 nodes in Fig.9(c). A similar trend of complete linearity in DoC can be observed when the density is further increased from 150-250. The outcome infers that there are two forms of density factor i.e. i) density within a limit and ii) density beyond a limit. Therefore, density within a limit is considered for 10-150 nodes and density beyond a limit is more than 150 nodes. This inference of the outcome also states that congestion may take place when there is jam of specific numbers of the vehicle that a particular segment of the road can accommodate i.e. Fig. 9(a)-Fig. 9(b). For example, if a segment of a lane can accommodate 70 vehicles so the maximum permissible limit of density can be only 70 here. In such case, the lower limit could be 20-40 nodes depending upon their speed limits.

However, considering the density of more than 150 is nearly impractical for a segment of a road in the real word, and hence the linearity behavior is shown in Fig.9(c) and Fig.9 (d). We carry out this analysis to testify the reliability of outcomes for proposed MIMC.

TABLE IV. NUMERICAL OUTCOME OF COMPARATIVE ANALYSIS

\begin{tabular}{|l|l|l|l|}
\hline & Delay & $\begin{array}{l}\text { Packet Delivery } \\
\text { Ratio }\end{array}$ & Throughput \\
\hline $\begin{array}{l}\text { Vadivel et al. } \\
{[10]}\end{array}$ & 5.28 & 1.53 & 1923 \\
\hline $\begin{array}{l}\text { Bhadauria et } \\
\text { al.[16] }\end{array}$ & 3.41 & 1.01 & 1200 \\
\hline AODV [28] & 7.85 & 2.47 & 1765 \\
\hline DSDV [29] & 7.26 & 3.12 & 1632 \\
\hline TORA [30] & 6.31 & 3.23 & 1771 \\
\hline Proposed & 1.89 & 5.55 & 4306 \\
\hline
\end{tabular}

The outcome of the proposed system is compared with multiple existing technique with respect to delay, packet delivery ratio, and throughput. The most work carried out by Vadivel et al. [10] is able to perform congestion control but the load balancing algorithm developed by them perform recursive operation of extracting Absolute Congestion Index from their neighbor nodes. The computation increases with increase in node density which was not considered in this study. Similarly work carried out by Bhadauria [16] uses mobile agents instead of middleware. The complete work is done by enhancing AODV itself which cannot contribute much to decision making of routing. Similar forms of problems also exists in conventional AODV, DSDV, and TORA also, which are overcome in proposed MIMC. Similar trend of packet delivery ratio and

Fig. 9. Impact of Density over DoC 
throughput can be observed, which shows that proposed system performs better congestion identification in contrast to the existing system. The primary reason behind this outcome is an inclusion of traffic modelling which uses multi-valued logic to accomplish better decision making and better inference to the state of congestion. The secondary reason behind the better outcome of MIMC is we have developed algorithm separately for identification and routing through alternative path which are less congested. This phenomenon results into execution of multiple operations at same time without involving much network related resources. MIMC can be used for any mobilebased adhoc application as well as vehicular application.

\section{CONCLUSION}

This paper has discussed the extension of our prior research work towards middleware design. We strongly believe that adoption of middleware in the hybrid mobile ad-hoc network is extremely important that can also cater up to the need of futuristic communication requirement. After reviewing existing literature, we find that there is few research work of middleware system focusing on addressing congestion problem while existing congestion control protocol doesn't consider middleware system. The proposed MIMC is meant to bridge this gap of research. The significant contribution of MIMC can be summarized as - i) the traffic modelling incorporated in MIMC is capable of representing multiple formats of speed and density to give better inference system. This is carried out to because existing congestion control protocols using frequently used AODV or OLSR lacks decision making for which reason the studies are limited to the offline investigation. ii) The control identification module is designed completely by a particular segment of the route (which represents a small part of any road in real-world). Hence, the applicability of the proposed system in real-world is quite high in the vehicular ad-hoc network. iii) We have not used any sophisticated optimization or mathematical modelling as we aimed to get better response time for any query toward extracting congestion information, iv) the mechanism of routing doesn't include any route acknowledgment, which minimized overhead to a larger extent if multicast protocols would be used here. Finally, the congestion mitigation is done by exploring routes which are less congested. Although the mechanism uses iterative principle, it is highly controlled by the rule-based control matrix. The study outcomes show that MIMC performs better congestion control as compared to the existing system.

\section{REFERENCES}

[1] A. Abdelaziz, M. Nafaa, G. Salim, "Survey of Routing Attacks and Countermeasures in Mobile Ad Hoc Networks", IEEE International Conference on Computer Modelling and Simulation, pp.693-698, 2013

[2] W. A. Jabbar, M. Ismail, R. Nordin, S. Arif, "Power-efficient routing schemes for MANETs: a survey and open Issues", Springer Journal of Wireless Networking, 2016

[3] D.K Anand, S. Prakash, "A Short Survey of Energy-Efficient Routing Protocols for Mobile Ad-Hoc Networks", IEEE International Advances in recent Technologies in Communication and Computing, pp.327-329, 2010

[4] D Maheshwari and R Nedunchezhian, "Load Balancing in Mobile Ad Hoc Networks: A Survey", International Journal of Computer Applications, vol.59, iss.16, pp.44-49, December 2012

[5] H. Gupta and P. Pandey, "Survey of routing base congestion control techniques under MANET," IEEE- International Conference on Emerg- ing Trends in Computing, Communication and Nanotechnology (ICECCN), pp. 241-244, 2013

[6] S. M. Mirhosseini and F. Torgheh, "ADHOCTCP: Improving TCP Performance in Ad Hoc Networks", IntechOpen, DOI: 10.5772/13510, 2011

[7] S. M. Adam, R. Hassan, "Delay aware Reactive Routing Protocols for QoS in MANETs: a Review", Elsevier-ScienceDirect Journal of Applied Research and Technology, vol.11, Iss.6., 2013

[8] G. Paroux, I. Demeure, D. Baruch, "A survey of middleware for mobile ad hoc networks", Département Informatique et Réseaux, 2007

[9] S. Bandyopadhyay, M. Sengupta, S. Maiti, and S. Dutta, "A Survey of Middleware for Internet of Things", Springer Journal of Recent Trends in Wireless and Mobile Networks, vol.162, pp.288-296, 2011

[10] R. Vadivel, V. M. Bhaskaran, "Adaptive reliable and congestion control routing protocol for MANET", Springer Journal of Wireless Network, 2016

[11] M.D. Sirajuddin, Ch. Rupa and A. Prasad, "Advanced Congestion Control Techniques for MANET", Springer Journal of Information Systems Design and Intelligent Applications, Advances in Intelligent Systems and Computing, vol.433, 2016

[12] D. V. Pashchenko, M. S. Jaafar, S. A. Zinkin, D. A. Trokoz, "Directly executable formal models of middleware for MANET and Cloud Networking and Computing", Journal of Physics: Conference Series, vol.710, 2016

[13] W. Chen, Q. Guan, S. Jiang, Q. Guan, and T. Huang, "Joint QoS provisioning and congestion control for multi-hop wireless networks", Springer- EURASIP Journal on Wireless Communications and Networking, 2016

[14] B.C. Sreenivas, G.C. Bhanu Prakash, and K.V. Ramakrishnan, "MADTCP: An Approach for Congestion Control in MANET", Springer Journal of Advances in Computing \& Inf. Technology, vol.178, pp.531540,2013

[15] C. Greco, M. Cagnazzo, B. P. Popescu, "Low-Latency Video Streaming With Congestion Control in Mobile Ad-Hoc Networks", IEEE Transactions on multimedia, vol. 14, no. 4, August 2012

[16] S. S. Bhadauria and V. K. Sharma, "Framework and Implimentation of an Agent Based Congestion Control Technique for Mobile Ad-hoc Network”, Springer Journal, vol.125, pp.318-327, 2011

[17] Z. Qin, L. Iannarioy, C. Giannelliz, P. Bellavista, "MINA: A Reflective Middleware for Managing Dynamic Multinetwork Environments", IEEE Network Operation and Management Symposium, 2014

[18] P. Kamisinski, V. Goebel, and T. Plagemann, "A reconfigurable distributed CEP middleware for diverse mobility scenarios", IEEE International Conference on Pervasive Computing and Communications Workshops, pp.615-620, 2013

[19] G. Denker, N. Dutt, S. Mehrotra, "Resilient dependable cyber-physical systems: a middleware Perspective", Springer Journal of Internet Service Application, vol.3, pp.41-49. 2012

[20] S.G. Pease, "ROAM: supporting safety critical applications in MANETs with cross-layer middleware", IEEE 14th International Symposium on aWorld of Wireless, Mobile and Multimedia Networks (WoWMoM), Madrid, Spain, pp.1-2, 2013

[21] P. G. Lopez, R. G. Tinedo, J. M. B. Alsina, "Moving routing protocols to the user space in MANET middleware", Elsevier-Journal of Network and Computer Applications, vol.33, pp.588-602, 2010

[22] S. Liu, "A Context-Aware Reflective Middleware Framework for Mobile Ad-hoc and Wireless Sensor Networks", Thesis of Lehigh University, 2012

[23] J.Y. Kim, G. S. Tomar, L. Shrivastava,, "Load Balanced Congestion Adaptive Routing for Mobile Ad Hoc Networks", International Journal of Distributed Sensor Networks, 2014

[24] D. W. Kum, W. K. Seo, J. I. Choi and Y. Z. Cho, "Mobility adaptive hybrid routing for mobile ad hoc networks," IEEE International Conference on Computer Science and Automation Engineering (CSAE), pp. 377-381, 2012

[25] M. U. Farooq and N. Tapus, "CEHR: Core enabled hybrid routing protocol for mobile ad hoc networks," IEEE International Conference on 
Intelligent Computer Communication and Processing, pp. 349-354, 2014

[26] P. G. S. Hiremath and C. V. G. Rao, "MERAM: Message exchange with resilient and adaptive middleware system in MANET," IEEE International Conference on Computational Intelligence and Computing Research (ICCIC), Madurai, pp. 1-6, 2015

[27] P. G. Sunitha Hiremath, C. V. Guru Rao, "MEEM: A Novel Middleware for Energy Efficiency in Mobile Adhoc Network", Springer Software Engineering Perspectives and Application in Intelligent Systems, vol.465, 2016
[28] Perkins, C.; Belding-Royer, E.; Das, S. Ad hoc On-Demand Distance Vector (AODV) Routing. IETF. RFC 3561. Retrieved 2010-06-18., 2003

[29] Perkins, Charles E.; Bhagwat, Pravin "Highly Dynamic DestinationSequenced Distance-Vector Routing (DSDV) for Mobile Computers" (pdf). Retrieved 2006-10-20, 2994

[30] V. D. Park and M. S. Corson. "A highly adaptive distributed routing algorithm for mobile wireless networks". In Proceedings of INFOCOM, 1997 